\begin{tabular}{|c|l|}
\hline Title & Efficient topological chaos embedded in the blinking vortex system \\
\hline Author(s) & Kin, Eiko; Sakajo, Takashi \\
\hline Citation & Hokkaido University Preprint Series in Mathematics, 669, 1-18 \\
\hline Issue Date & 2004 \\
\hline DOI & 10.14943/83820 \\
\hline Doc URL & http://hdl.handle.net/2115/69474 \\
\hline Type & bulletin (article) \\
\hline File Information & pre669.pdf \\
\hline
\end{tabular}

Instructions for use 


\title{
Efficient topological chaos embedded in the blinking vortex system
}

\author{
Eiko KIN \\ Department of Mathematics, Kyoto University, \\ Kitashirakawa Oiwake-cho Sakyo-ku, Kyoto 606-8502 JAPAN, \\ Tel: +81-75-753-3698, Fax: +81-75-753-3711, \\ E-mail: kin@math.kyoto-u.ac.jp \\ and \\ Takashi SAKAJO \\ Department of Mathematics, Hokkaido University, \\ Kita 10 Nishi 8 Kita-ku, Sapporo 060-0810 JAPAN, \\ Tel: +81-11-706-4660, Fax: +81-11-727-3705, \\ E-mail: sakajo@math.sci.hokudai.ac.jp
}

\begin{abstract}
Periodic orbits for homeomorphisms on the plane give mathematical braids, which are topologically classified into three types by Thurston-Nielsen (T-N) theory; (1) periodic, (2) reducible, and (3) pseudo-Anosov (pA). If the braid is $\mathrm{pA}$, then the homeomorphism must have an infinitely many number of periodic orbits of distinct periods. This kind of complexity induced by the pA braid is called "topological chaos", which was introduced by Boyland et. al [4] recently. We investigate numerically the topological chaos embedded in the particle mixing by the blinking vortex system introduced by Aref [1]. It has already been known that the system generates the chaotic advection due to the homoclinic chaos, but the chaotic mixing region is restricted locally in the vicinity of the vortex points. In the present study, we propose an ingenious operation of the blinking vortex system that defines a mathematical braid of $\mathrm{pA}$ type. The operation not only generates the chaotic mixing region due to the topological chaos, but also ensures global particle mixing in the whole plane. We give a mathematical explanation for the phenomenon by the T-N theory and some numerical evidences to support the explanation. Moreover, we make mention of the relation between the topological chaos and the homoclinic chaos in the blinking vortex system.
\end{abstract}

Keywords: Topological chaos, blinking vortex, chaotic advection, Thurston-Nielsen theory, braids

\section{Introduction}

For a given velocity field $\mathbf{u}(\mathbf{x}, t)$ in $\mathbf{x} \in \mathbb{R}^{3}$ and $t \in \mathbb{R}$, a light and inert particle (passive scalar) is advected by the following ordinary differential equation:

$$
\frac{d \mathbf{x}_{p}}{d t}=\mathbf{u}\left(\mathbf{x}_{p}, t\right), \quad \mathbf{x}_{p}(0)=\mathbf{x}_{p 0},
$$

in which $\mathbf{x}_{p}(t) \in \mathbb{R}^{3}$ represents the position of the passive scalar. The evolution of the velocity field $\mathbf{u}$ is described by the fluid equations such as the Navier-Stokes 
equations, the Euler equations and the Stokes equations. The advection equation gives the Lagrangian description of the motion of fluid particles. When the particles show chaotic behavior, we call it "the chaotic advection". The topic has been attracting many researchers for the last several decades, since the chaotic advection is observed even if the flow itself exhibits no chaotic behavior. A brilliant survey in terms of the development of the topic is given by Aref [2].

When we focus on the two-dimensional incompressible flows in particular, the velocity field is derived from a stream function, say $\Psi$, as follows.

$$
\mathbf{u}=\left(\frac{\partial \Psi}{\partial y},-\frac{\partial \Psi}{\partial x}\right)
$$

Hence, the equation of motion for the passive scalar located at $\mathbf{x}_{p}=\left(x_{p}, y_{p}\right)$ is given by

$$
\frac{d x_{p}}{d t}=\frac{\partial \Psi}{\partial y}, \quad \frac{d y_{p}}{d t}=-\frac{\partial \Psi}{\partial x} .
$$

The advection equations are the Hamiltonian dynamics, whose Hamiltonian is equivalent to the stream function. When the stream function is time-independent, the particles show no chaotic behavior, since it is integrable. On the other hand, however, the time-dependent Hamiltonian dynamical system could generate the chaotic advection. The theory of dynamical systems explains that the phenomenon occurs when the stable and the unstable manifolds of the hyperbolic fixed points for the flow cross transversely, which is known as the homoclinic chaos.

On the other hand, Boyland et al.[4] introduced the another notion of topological chaos for the chaotic advection. They considered particle mixing experimentally by three batch stirrers in a two-dimensional disk filled with viscous fluid, called a batch stirring device, and observed the chaotic particle mixing when the batch stirrers are moved in some specific way. To explain the chaotic mixing in the batch stirring device, they adopt Thurston-Nielsen theory (the T-N theory), which indicates that any homeomorphism on a two-dimensional manifold is isotopic to one of the following maps; periodic map, pseudo-Anosov map ( $p A$ map) and reducible map. The periodic map generates simple dynamics, since it is essentially equivalent to a rotation map. On the other hand, the pA map generates chaotic dynamics, because it is similar to Smale's horseshoe map. That is, the map stretches uniformly in one direction and contracts uniformly in another direction at every point. The reducible map means that it can be decomposed into some restricted maps, each of which is isotopic to either a periodic map or a pA map. We say that the isotopy class of a given homeomorphism $f$ is periodic, $p A$ and reducible, if $f$ is isotopic to a periodic map, a pA map, and a reducible map respectively. As for the details of the theory, see $[7,15]$, and a survey paper [5].

Chaotic dynamics generated by the pA map $\Phi$ is essentially embedded in the dynamics of any map that is isotopic to $\Phi$. In fact, M. Handel [11] shows that if a homeomorphism $f: M \rightarrow M$ on a two-dimensional manifold $M$ is isotopic to a pA map $\Phi: M \rightarrow M$, then there exist a compact $f$-invariant set $Y \subset M$ and a continuous and surjective map $g: Y \rightarrow M$ such that $\left.g \circ f\right|_{Y}=\Phi \circ g$. In other words, chaotic dynamics of the pA map remains after continuous deformation of $\Phi$. Generally speaking, since $g$ is many to one map, the dynamics of $f$ is more complicated than that of $\Phi$. Boyland et al. [4] pointed out that the motion of the batch stirrers defined a braid that specifies some isotopy class on the punctured disk. Therefore, if the braid is $\mathrm{pA}$, it turns out that the batch stirring device generates the complexity forced by the pA braid. They defined the term, topological chaos, as chaotic dynamics forced by the pA map $\Phi$ in the sense of Handel's theorem.

An important property of the particle mixing due to the chaotic advection is how efficient the particles are stirred in the chaotic mixing region. Generally speaking, 
the chaotic region generated by the homoclinic chaos contains island structures generated by the collapse of KAM tori. Thus the particles move periodically in the local island structures, while those outside the islands moves disorderly. In this sense, we observe the non-uniform chaotic mixing. On the other hand, in the chaotic region generated experimentally by the topological chaos [4], the particles are stirred quite uniformly. Hence, it seems that the topological chaos results in the efficient particle mixing than the homoclinic chaos. However, in the meantime, it is mathematically shown that the topological chaos forced by the pA braid is embedded in the homoclinic chaos [9]. It indicates that a uniform chaotic mixing region due to the topological chaos is contained somewhere in the non-uniform chaotic mixing region due to the homoclinic chaos.

Based on the above consideration, we are going to deal with the particle mixing by the blinking vortex model that Aref [1] introduced. The model is not the Hamiltonian system because of the temporal discontinuity of the velocity field and it is known that the particle mixing due to the homoclinic chaos occurs. See for the details in Chapter 7.3 of Ottino's textbook [14]. We investigate numerically how the topological chaos is embedded in the homoclinic chaos in the mixing process. Thus we propose a more efficient mixing mechanism by a controlled operation of the blinking vortex device under consideration of the T-N theory.

The paper consists of five sections. In $\S 2$, we introduce our mixing device by the blinking vortices and explain how to operate it. Then, we give numerical examples of the particle mixing by two different operations of the mixing device, and point out remarkable differences in the chaotic mixing in $\S 3$. In $\S 4$, we suggest a conjecture that explains the differences with the help of the T-N theory and show some numerical evidences supporting the conjecture. Last section is devoted to the conclusion.

\section{Blinking vortex system and its operation}

We consider an imaginary mixing device in two-dimensional space, in which two mixing agitators, say $P_{1}$ and $P_{2}$, are installed. It is possible to control the direction and the duration of the rotation of the agitators precisely. Suppose that each of the agitators generates a vortex point regularized by the vortex method, called a "vortex blob", the strength of which is given by 1 when the agitator rotates counterclockwise, and by -1 when it rotates clockwise. We also assume that when the agitator begins rotating, the vortex blob develops instantly and then particles are stirred. On the other hand, when the agitator stops, the vortex blob disappears and the particles also stop immediately.

Let $\left(p_{i}, q_{i}\right)$ represent the position of the agitator $P_{i}$ for $i=1,2$. Then, the vortex blob generated by $P_{i}$ induces the following velocity field $\left(u_{i}, v_{i}\right)$ :

$$
\begin{aligned}
& u_{i}(x, y)=-\frac{\Gamma_{i}}{2 \pi} \frac{y-q_{i}}{\left(x-p_{i}\right)^{2}+\left(y-q_{i}\right)^{2}+\delta^{2}}, \\
& v_{i}(x, y)=\frac{\Gamma_{i}}{2 \pi} \frac{x-p_{i}}{\left(x-p_{i}\right)^{2}+\left(y-q_{i}\right)^{2}+\delta^{2}},
\end{aligned}
$$

in which $\Gamma_{i}$, which is either 1 or -1 , represents the strength of the vortex blob and the positive $\delta$ is the regularization parameter of the vortex method. When $\delta$ is exactly zero, the flow (1) and (2) corresponds to the one induced by a vortex point in the incompressible and inviscid fluid, which diverges as $(x, y)$ tends to $\left(p_{i}, q_{i}\right)$. In order to avoid the singularity, introducing $\delta \neq 0$ gives a regularized velocity field. The regularization method is often used in the numerical studies of the inviscid and incompressible flows [12]. Mathematically speaking, it is shown that for sufficiently small $\delta$, the vortex method gives an accurate approximation for the inviscid flows 
as long as they are smooth [6]. Another numerical example shows that this method also effectively approximates the incompressible Navier-Stokes equations with small viscosity [13].

Here, let us explain the specifications of the mixing device used in the paper. The two agitators $P_{1}$ and $P_{2}$ are located at $\left(-\frac{1}{2}, 0\right)$ and $\left(\frac{1}{2}, 0\right)$ respectively. Once the agitator $P_{1}$ is switched on, it continues to rotate until the particles at the positions $(-1,0)$ and $(0,0)$ are interchanged mutually. Hence, it follows from (1) and (2) that the duration of the rotation $P_{1}$ is given by

$$
T=2 \pi^{2}\left(\delta^{2}+\frac{1}{4}\right)
$$

In the same way, the vortex blob generated by $P_{2}$ interchanges the particles at $(0,0)$ and $(1,0)$ by rotating for the same time period $T$. Moreover, we operate the two agitators exclusively. That is to say, when the agitator $P_{1}$ is active, the agitator $P_{2}$ must be inactive and vice versa.

We are going to observe numerically how particles spread when the vortex blobs generated by $P_{1}$ and $P_{2}$ are blinking periodically. Then the ordinary differential equations for $N$ particles are given as follows,

$$
\begin{aligned}
\frac{d x_{p}}{d t} & =u_{1}\left(x_{p}, y_{p}\right) \chi_{1}(t)+u_{2}\left(x_{p}, y_{p}\right) \chi_{2}(t) \\
\frac{d y_{p}}{d t} & =v_{1}\left(x_{p}, y_{p}\right) \chi_{1}(t)+v_{2}\left(x_{p}, y_{p}\right) \chi_{2}(t)
\end{aligned}
$$

for $1 \leq p \leq N$, in which the function $\chi_{i}(t)$ is defined by

$$
\chi_{i}(t)= \begin{cases}1 & \text { if } P_{i} \text { is active } \\ 0 & \text { if } P_{i} \text { is inactive. }\end{cases}
$$

In what follows, for the sake of easy description of the mixing process, when the agitator $P_{i}$ rotates counterclockwise for the time period $T$, the operation is denoted by $p(i)$. On the contrary, when $P_{i}$ stirs the particle clockwise, it is symbolized by $n(i)$.

The forth-order Runge-Kutta method is used for the numerical integration of (3) and (4). The step size of the temporal discretization is $\Delta t=0.001 \times T$. At the initial moment, the particles distribute uniformly in the segment $[-2,2]$, which are represented by

$$
x_{p}(0)=-2.0+4.0 \frac{p}{N}, \quad y_{p}(0)=0, \quad \text { for } 1 \leq p \leq N .
$$

The number of the particles $N$ equals 20000 .

\section{Chaotic advection by the blinking vortex blobs}

First, we observe the mixing of the particles when we repeat the operation $p(1) n(2)$. In Figure 1, we plot the distribution of the particles every two operations of $p(1) n(2)$, i.e. at $t=k T$ for $k=4,8, \cdots, 32$. The regularization parameter $\delta$ is 0.6 . The symbols $\bigcirc, \square$ and $\triangle$ in the figure represent the positions $(-1,0),(0,0)$ and $(1,0)$ respectively.

Initially, the particles distribute uniformly in the segment $[-2,2]$. When the operation $p(1) n(2)$ is done twice, there appear streaks of the particles between $\bigcirc$ and $\triangle$ over $\square$. After four operations, while the number of the streaks increases, the right tip of the outermost streak approaches $\square$ passing the right-hand side of $\triangle$. At the sixth operation, there emerge other streaks of particles going from $\square$ to 
by passing the right-hand side of $\triangle$ and above $\square$. As the number of the operations increases, the two streaks fill in a region densely and the particles finally spread uniformly in the region.

We observe the dynamics of the particles in the mixing process. Figure 2 shows the orbits of the ten particles $\left(x_{i}, y_{i}\right)$ for $i=0,2000,4000, \cdots, 18000$ when we repeat the operation $p(1) n(2)$ many times. The orbits consist of a disorderly mixing region in the neighborhood of the vortex blobs and many return orbits connecting between the top and the bottom of the disorder region. In order to see how the particles spread in detail, we plot the evolution of $x$-coordinates of the sample particles $\left(x_{i}, y_{i}\right)$ for $i=406,407,10104,10105,19896$ and 19897 for the first 30 operations in Figure 3. Initially, the distances between the adjoining two particles are sufficiently small, i.e. $\frac{1}{N}=5.0 \times 10^{-5}$ due to (5), and the orbits of the two particles are quite close up to the 10th operation. However, from the 10th through the 20th operations, during which all the sample particles exist in the disorder region, their orbits separate considerably and fluctuate disorderly. This indicates that the mixing process is sensitive to the initial positions of the particles. In this sense, the particles are stirred chaotically in the disorder region. After the 20th operation, some of them go out of the chaotic mixing region and then move along the return orbits, while the others remain in the chaotic region. Since the particles going out of the chaotic region eventually return to the chaotic region along the return orbits, they are stirred chaotically again. In other words, every single particle shows intermittent chaotic behavior. We note that the intermittent dynamics guarantees that all the particles in $\mathbb{R}^{2}$ are stirred chaotically, since any particle, however far it is from the vortex blobs, necessarily enters the chaotic region after moving along the return orbits.

Now, we are going to describe the structure of the particles embedded inside the chaotic mixing region observed in Figure 1. See Figure 4 for the schematic reference:

(1) With the first operation, the initial line segment of the particles is stretched and folded counterclockwise, and as a result it forms a streak going from $\bigcirc$ to $\triangle$ and then back $\bigcirc$ over $\square$, which we call a "○-streak". The next operation maps the $\bigcirc$-streak outward and generates a new $\bigcirc$-streak inside the old $\bigcirc$-streak. It indicates that the operation expands the $\bigcirc$-streaks outward, while generating a new $\bigcirc$-streak inside them. See the distributions at the second and the fourth operations in Figure 1 for comparison.

(2) The right tip of the outermost $\bigcirc$-streak approaches $\square$ passing the right-hand side of $\triangle$. Then, it is stretched and folded clockwise and forms another streak of particles, which goes from $\square$ to $\bigcirc$ by passing the right-hand side of $\triangle$ and over $\square$ and returns on its way back to $\square$. We refer to the new streak as the " $\square$-streak". The $\square$-streaks are also expanded outward by the operation with the generation of a new $\square$-streak in the same way as the $\bigcirc$-streaks.

(3) As the number of the operations increases, the $\square$-streaks penetrate alternately between the $\bigcirc$-streaks. The left tips of the both streaks in the neighborhood of $\bigcirc$ are caught in the innermost $\bigcirc$-streak as we see in the distributions of the 10th and 12th operations in Figure 1. On the other hand, the right tips of the both streaks concentrate in $\square$. Thus, the repetition of the operation results in formation of the alternate stripe structure of the $\bigcirc$-streaks and the $\square$-streaks, which is like a horseshoe structure.

Next, we show the mixing of the particles by repeating the operation $p(1) n(2)$ for $\delta=0.2$ and $\delta=0.4$ in Figure 5. Since the flow tends to be inviscid as $\delta$ decreases, the velocity field in the neighborhood of the vortex blobs is getting less regular, which results in the strong stirring around the vortex blobs. However, the chaotic mixing is finally observed after the formation of the horseshoe-like structure. On the other hand, the chaotic region develops more quickly as $\delta \rightarrow 0$, since the less 

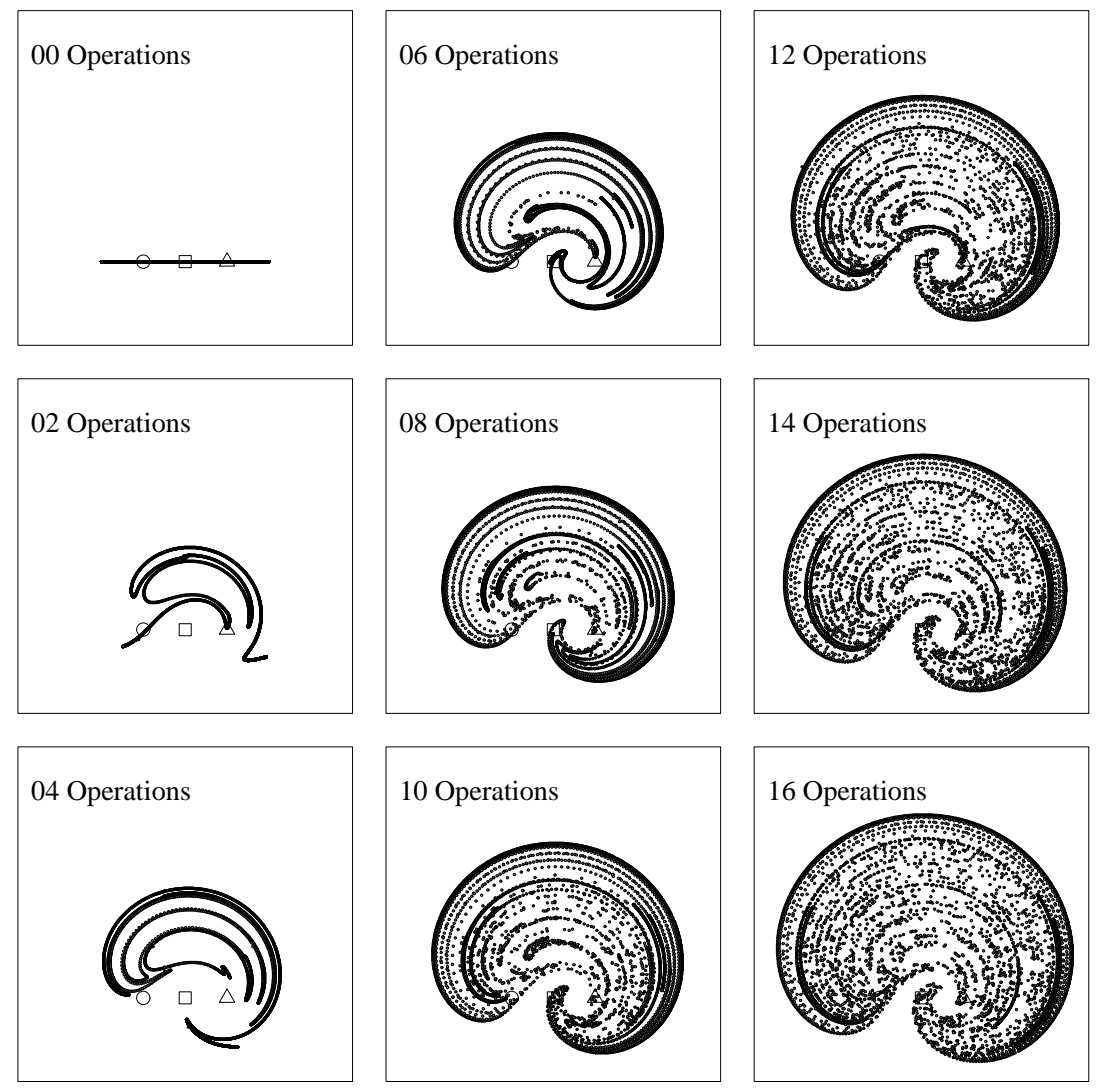

Figure 1: Mixing of the particles when the operation $p(1) n(2)$ is repeated. The regularization parameter $\delta$ is 0.6 . 


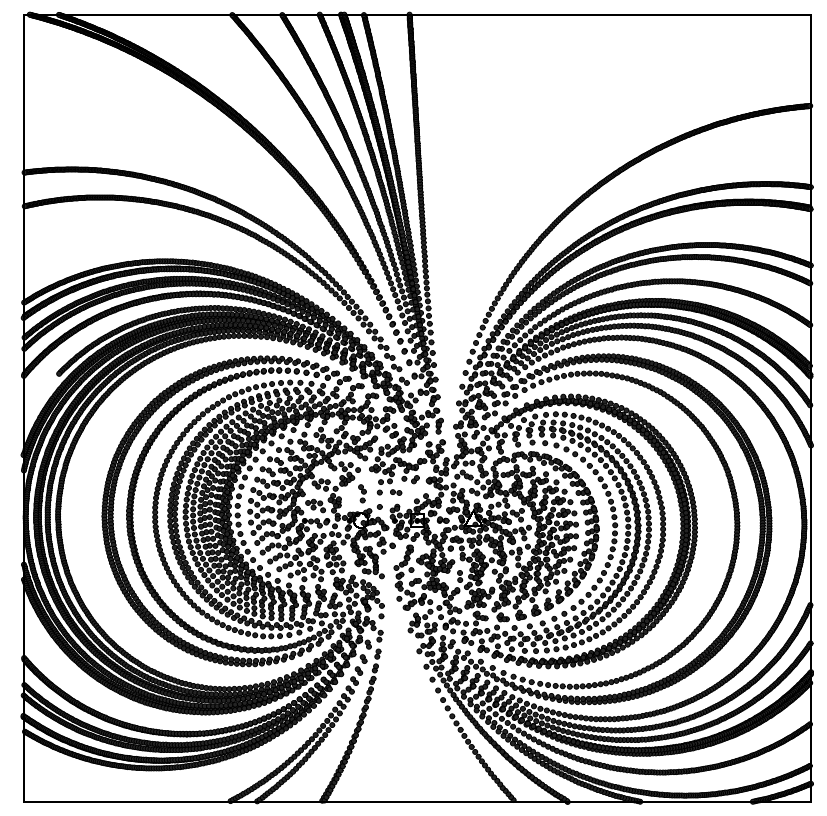

Figure 2: The orbits of the ten particles, $\left(x_{i}, y_{i}\right)$ for $i=0,200,400, \cdots, 1800$ stirred by the operation $p(1) n(2)$ many times. There exist a disorderly mixing region in the neighborhood of the vortex blobs and many orbits that depart and return again to the region.

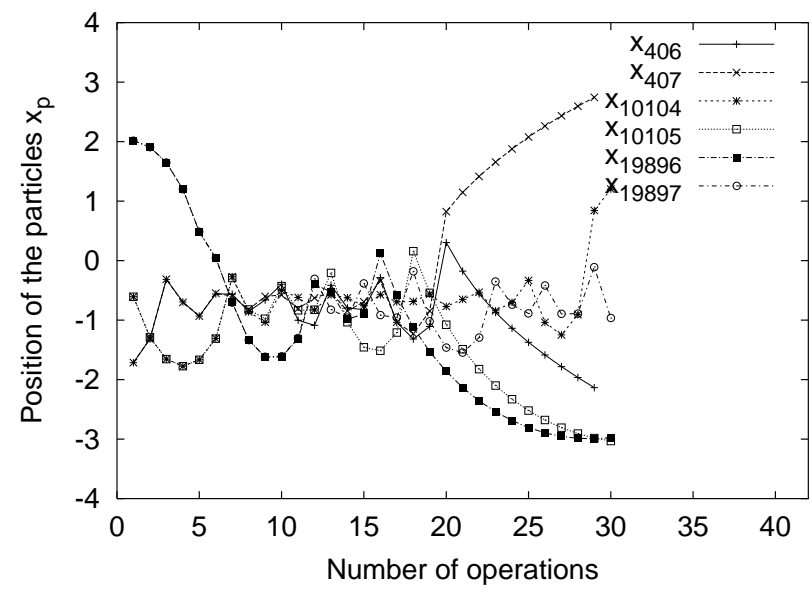

Figure 3: Evolution of the $x$-coordinates of the sample particles. The orbits of the two adjoining particles separate from each other after the 10th operations, although the initial distance between them is sufficiently small. 


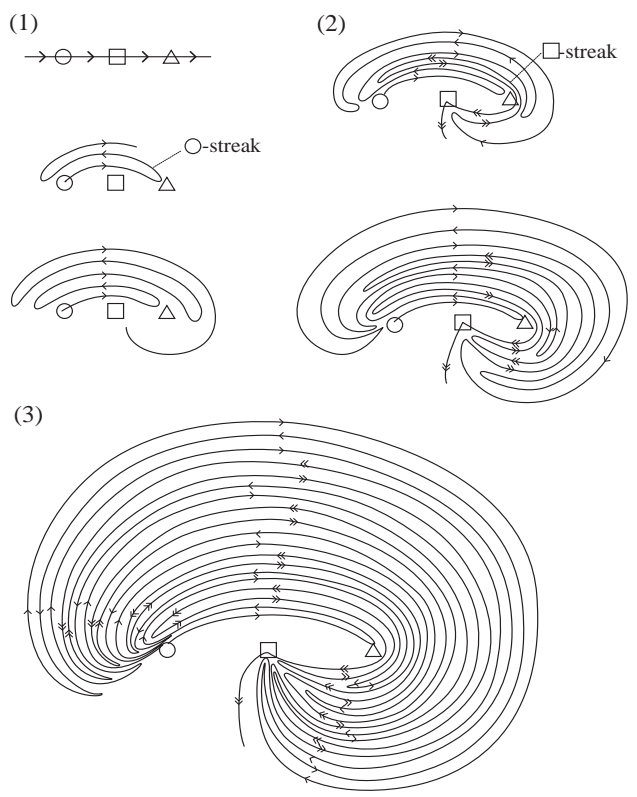

Figure 4: Horseshoe-like structure embedded in the chaotic region.

viscous flow stirs the particles more strongly.

Finally, we show the particle mixing by the other operation $p(1) p(2)$ for $\delta=0.6$ in Figure 6. The initial straight segment of the particles winds around the vortex blobs as the number of the operations increases. No chaotic region as we have observed in Figure 1 appears, though there exists a small disorder region in the neighborhood of the vortex blobs. Figure 7 shows the orbits of the ten particles $\left(x_{i}, y_{i}\right)$ for $i=0,200,400, \cdots, 18000$ when we repeat the operation $p(1) p(2)$ many times. There certainly exists a small disorderly mixing region in the neighborhoods of the vortex blobs. However, the mixing region contains the island structures and the particles spread non-uniformly in the region. On the other hand, the particles outside the mixing region follow the rotational orbits around the region, and consequently they never enter the mixing region, which indicates that all the particles in $\mathbb{R}^{2}$ are hardly mixed chaotically unlike the case for the operation $p(1) n(2)$. Therefore, the operations $p(1) n(2)$ stirs the particles more efficiently than the operation $p(1) p(2)$ does, although the same amount of energy for the operations of the agitators is required.

\section{Braid dynamics and topological mixing}

\subsection{Pseudo-Anosov braids and topological mixing}

To understand the difference between the particle mixings by two operations $p(1) n(2)$ and $p(1) p(2)$, we apply the T-N theory. We first define homeomorphisms $F_{p(i)}$ and $F_{n(i)}: \mathbb{R}^{2} \rightarrow \mathbb{R}^{2}$ for $i=1,2$ as follows: Take a set of three points $A=\left\{a_{1}, a_{2}, a_{3}\right\}$ that lies in the $x$-axis in the plane, say $a_{1}, a_{2}, a_{3}$ from left to right. Let $F_{p(i)}$ (resp. $\left.F_{n(i)}\right)$ be a homeomorphism satisfying the following conditions: 
(a) delta $=0.2$
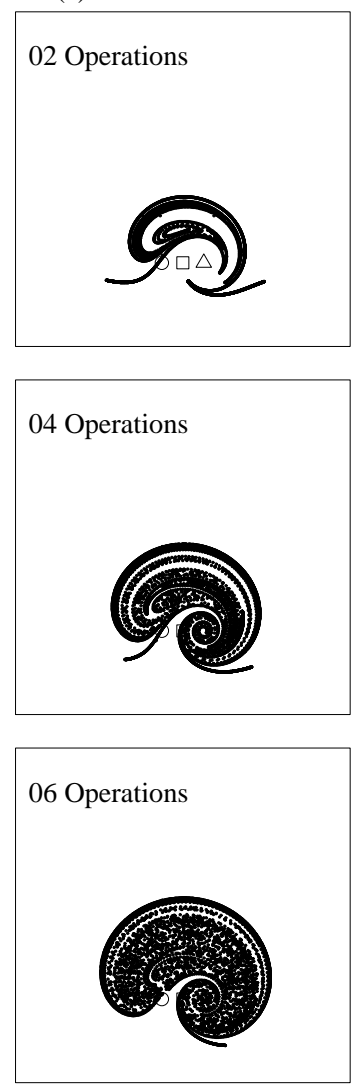

(b) delta $=0.4$
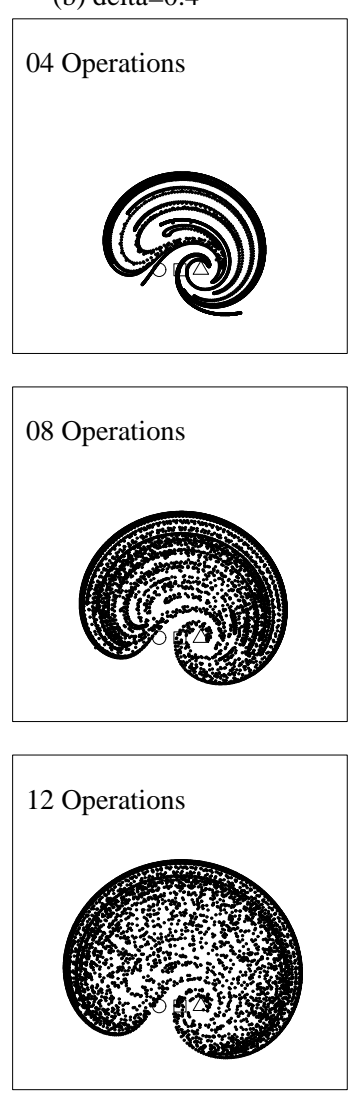

Figure 5: Mixing of the particles when the operation $p(1) n(2)$ is carried out repeatedly. The regularization parameters are (a) $\delta=0.2$ and (b) $\delta=0.4$. 

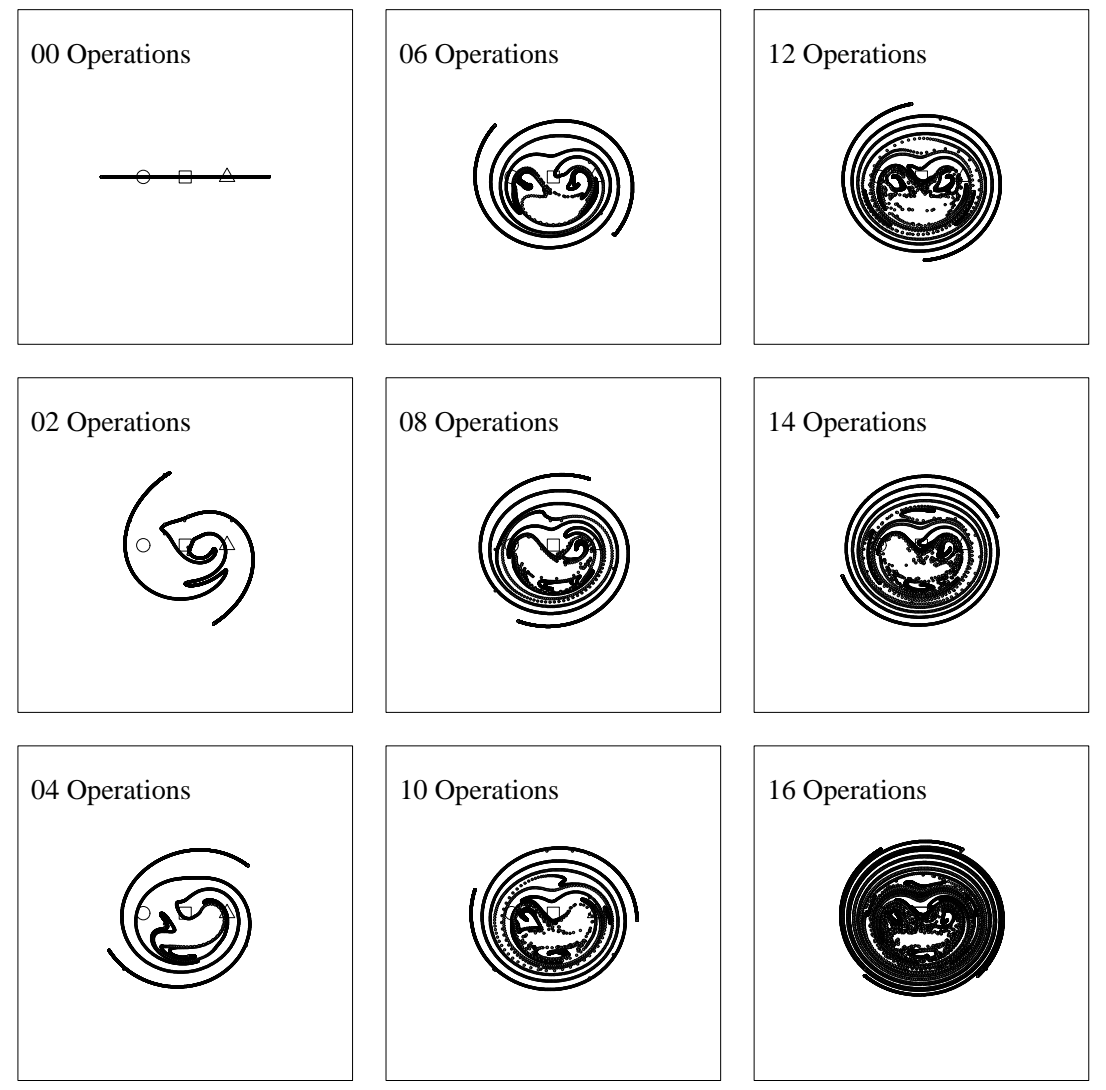

Figure 6: Mixing of the particles when we repeat the operation $p(1) p(2)$. The regularization parameter $\delta$ is 0.6 . 


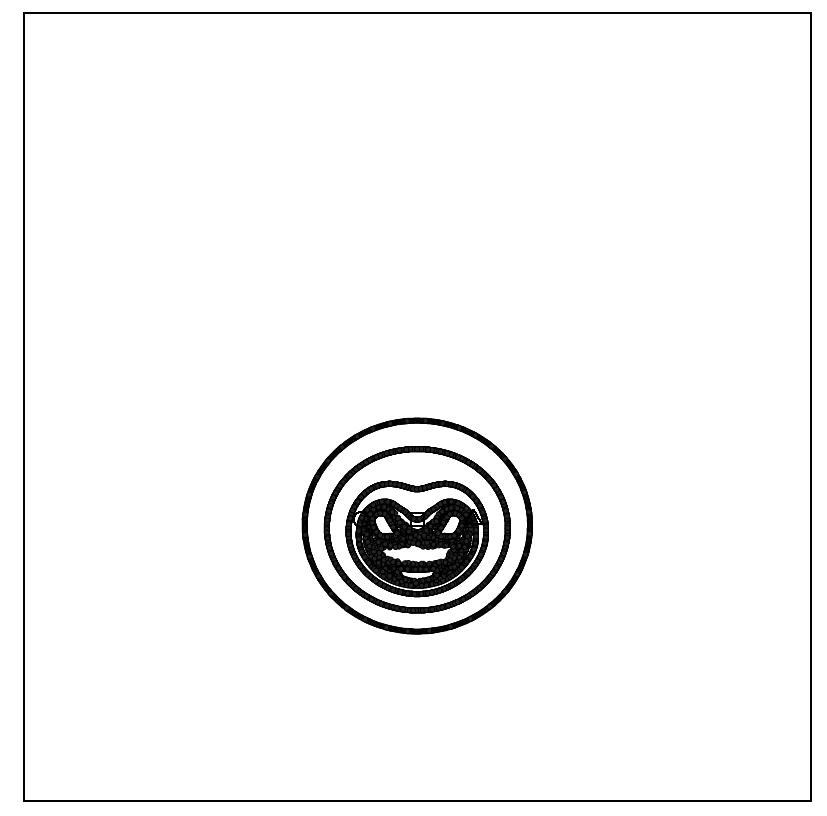

Figure 7: The orbits of the ten mixing particles, $\left(x_{i}, y_{i}\right)$ for $i=0,200,400, \cdots, 1800$ due to the operation $p(1) p(2)$. While there exists a disorderly mixing region in the neighborhood of the vortex blobs, the particles outside the disorder region follow the rotational orbits.

- Two points $a_{i}$ and $a_{i+1}$ are interchanged counterclockwise under $F_{p(i)}$

(resp. clockwise under $F_{n(i)}$ ) as in Figure 8, and

- the other point in $A$ is fixed under $F_{p(i)}\left(\right.$ resp. $\left.F_{n(i)}\right)$.

Since $A$ is invariant under $F_{p(i)}$ and $F_{n(i)}$, they are thought as the homeomorphisms on the 3-punctured plane $R_{3}=\mathbb{R}^{2} \backslash A$.

In general, for any homeomorphism $f: \mathbb{R}^{2} \rightarrow \mathbb{R}^{2}$ with a finite invariant set $P$, the isotopy class of $f$ on $\mathbb{R}^{2} \backslash P$ is specified by a braid on $\mathbb{R}^{2}$. We say that the braid is periodic, $p A$ and reducible, if the isotopy class of $\left.f\right|_{\mathbb{R}^{2} \backslash P}: \mathbb{R}^{2} \backslash P \rightarrow \mathbb{R}^{2} \backslash P$ is periodic, pA and reducible respectively. Accordingly, since the set $A$ is a period 3 orbit for $F_{p(1)} \circ F_{n(2)}$ and $F_{p(1)} \circ F_{p(2)}$, the homeomorphisms on $R_{3}$ are specified by some 3-braids on $\mathbb{R}^{2}$; Let $\sigma_{1}$ and $\sigma_{2}$ be the Artin's generators of the 3-braid group on $\mathbb{R}^{2}$ as in Figure 9. Then the isotopy class of $F_{p(i)}\left(\right.$ resp. $\left.F_{n(i)}\right)$ on $R_{3}$ is specified by the 3 -braid $\sigma_{i}$ (resp. $\sigma_{i}^{-1}$ ), since $F_{p(i)}$ interchanges $a_{i}$ and $a_{i+1}$ counterclockwise (resp. clockwise). Thus the 3-braids $\sigma_{1} \sigma_{2}^{-1}$ and $\sigma_{1} \sigma_{2}$ specify the isotopy classes of $F_{p(1)} \circ F_{n(2)}$ and $F_{p(1)} \circ F_{p(2)}$ on $R_{3}$ respectively. Note that the braids $\sigma_{1} \sigma_{2}^{-1}$ and $\sigma_{1} \sigma_{2}$ are $\mathrm{pA}$ and periodic respectively.

On the other hand, the operations $p(i)$ and $n(i)$ also determine homeomorphisms on $\mathbb{R}^{2}$, say $\widetilde{F}_{p(i)}$ and $\widetilde{F}_{n(i)}$. Now, we consider a relation between the maps $F_{p(i)}$ and $F_{n(i)}$, and the maps $\widetilde{F}_{p(i)}$ and $\widetilde{F}_{n(i)}$. Let $b_{1}, b_{2}$ and $b_{3}$ denote the positions $(-1,0),(0,0)$ and $(1,0)$ respectively. We compare the motion of the set of three points $B=\left\{b_{1}, b_{2}, b_{3}\right\}$ under $\widetilde{F}_{p(1)}$ with that of the set $A$ under $F_{p(1)}$. The map $\widetilde{F}_{p(1)}$ interchanges $b_{1}$ and $b_{2}$ counterclockwise in the same way as $F_{p(1)}$ does. Hence, $\widetilde{F}_{p(1)}\left(b_{1}\right)=b_{2}$ and $\widetilde{F}_{p(1)}\left(b_{2}\right)=b_{1}$, and the set $\left\{b_{1}, b_{2}\right\}$ gives an exact 2-braid, see Figure 10. A difference between $F_{p(1)}$ and $\widetilde{F}_{p(1)}$ is the motions of $a_{3}$ and $b_{3}$. While $a_{3}$ is fixed under $F_{p(1)}$, the point $b_{3}$ moves slightly due to $\widetilde{F}_{p(1)}$ as we show in 


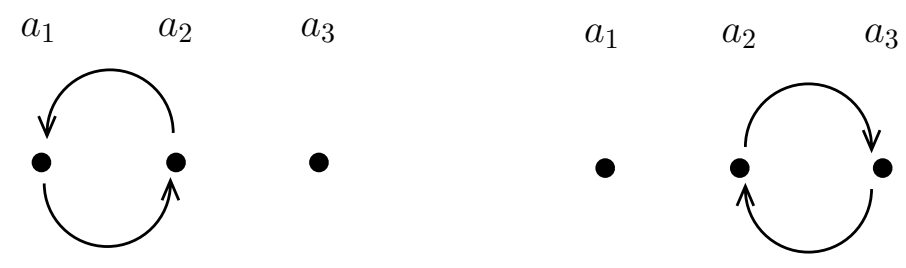

Figure 8: $F_{p(1)}[\mathrm{left}] ; F_{n(2)}[$ right $]$.
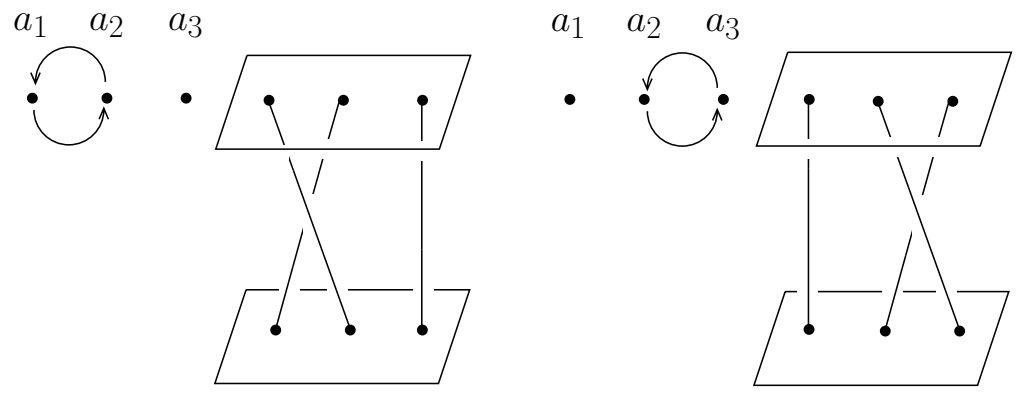

Figure 9: The Artin's generators $\sigma_{1}[\mathrm{left}]$ and $\sigma_{2}$ [right].

Figure 10. Specifically, $\widetilde{F}_{p(1)}$ advects $b_{3}$ counterclockwise around the agitator $P_{1}$ at $\left(-\frac{1}{2}, 0\right)$ by the angle $\theta(\delta)$,

$$
\theta(\delta)=\frac{1}{r} v_{1}\left(b_{3}\right) \times T,
$$

in which $r=\frac{3}{2}$ is the distance between the agitator $P_{1}$ and $b_{3}$. For example, $\theta(0.6) \approx 0.23 \pi$. It means that the set $B$ is not invariant under the homeomorphisms $\widetilde{F}_{p(i)}$ and $\widetilde{F}_{n(i)}$. Therefore, the homeomorphisms do not give exact $(2+1)$-braids. However, since $\theta(\delta)$ is insignificant for small $\delta$, the motion of the set $A$ under $F_{p(1)}$ approximates that of $B$ under $\widetilde{F}_{p(1)}$ topologically as in Figure 10. Hence, we assign the 3 -braids $\sigma_{i}$ and $\sigma_{i}^{-1}$ approximately to $\widetilde{F}_{p(i)}$ and $\widetilde{F}_{n(i)}$ respectively.

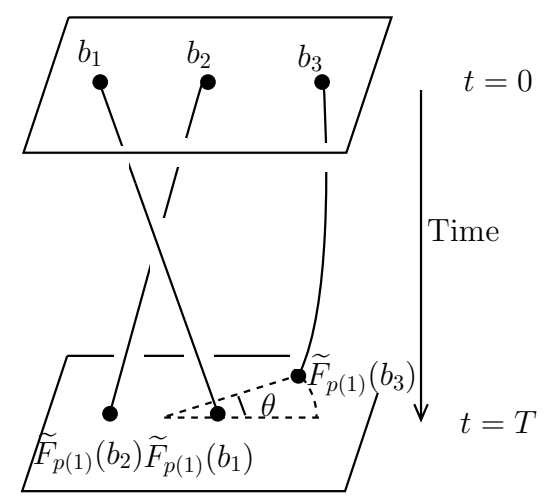

Figure 10: The motion of $B$

Thus the pA braid $\sigma_{1} \sigma_{2}^{-1}$ is topologically assigned to $\widetilde{F}_{p(1)} \circ \widetilde{F}_{n(2)}$. We now recall the chaotic region observed in Figure 1 and Figure 5 with a horseshoe-like structure 
(see also Figure 4) that fills densely in the neighborhood of the vortex blobs. It is remarkable that the strips structure of streaks in the region resembles the unstable manifold of the pA map on the 3-punctured plane, and that the shape of the chaotic region is also similar to the chaotic region shown by the laboratory experiment [4] and the numerical experiments $[8,16]$, which are examples of the topological chaos forced by the pA braid $\sigma_{1} \sigma_{2}^{-1}$. Furthermore, even if the initial particles distribute in the different positions, the similar horseshoe-like structure of streaks is observed as the number of the operations increases, since the particles eventually enter the chaotic mixing region after moving along the return orbits.

On the other hand, in the mixing process due to the operation $p(1) p(2)$, there is no horseshoe structure of streaks in the neighborhood of the vortex blobs, and the particles spread non-uniformly. The small disorderly mixing region observed in Figure 6 occurred by the homoclinic chaos in the neighborhood of the vortex blobs. In theory, the topological chaos is embedded in the homoclinic chaos somewhere in the region [9]. However, since the cause of the topological chaos is unrelated to the braid assigned for the operation, the region where the topological chaos exists occupies a small position in the whole chaotic region.

Finally, we make a conjecture which connects the assigned braid for the operation and the topological chaos; The topologically assigned braid for the operation determines the property of the topological chaos in the neighborhood of the vortex blobs; If the assigned braid is $\mathrm{pA}$, then the global chaotic region caused by the topological chaos occurs in the neighborhood of the vortex blobs. The assigned braid contributes to the occurrence of the global chaotic region. On the other hand, if the assigned braid is periodic, then no global chaotic region caused by the topological chaos occurs in the neighborhood of the vortex blobs. A local chaotic region might occur, but the assigned braid make no contribution to that chaotic region.

\subsection{Supporting numerical evidences for the conjecture}

\subsubsection{Particle mixing by the other operations}

Our conjecture given in the previous section is based on the observation of the particle mixing by the two operations $p(1) n(2)$ and $p(1) p(2)$. In order to see it is not the specific to the two cases, we show in Figure 11 the particle mixings by the operations $p(1) p(1) n(2) n(2)$ and $p(1) p(1) p(2)$, which are assigned approximately to the pA braid $\sigma_{1} \sigma_{1} \sigma_{2}^{-1} \sigma_{2}^{-1}$ and the periodic braid $\sigma_{1} \sigma_{1} \sigma_{2}$ respectively. While the similar expanding horseshoe-like structure appears in the former case, there emerges just a local chaotic region in the latter case. This indicates that the complexity forced by the other pA braid corresponding to the operator $p(1) p(1) n(2) n(2)$ is also embedded in the mixing process, which support the conjecture.

\subsubsection{Expansion constant and stretching rate}

There is an important quantity to characterize the chaotic region forced by the $\mathrm{pA}$ map; In the horseshoe-like region generated by the topological chaos, the pA map stretches uniformly in one direction at the rate $\lambda>1$ and contracts uniformly in another direction at the rate $1 / \lambda$ for every point. The number $\lambda>1$ is called the expansion constant. This constant gives how two points that are very close to each other initially are stretched by the pA map. To obtain the expansion constant for the pA maps, the Bestivina-Handel algorithm [3] or the software "Train tracks of surface homeomorphisms" [10] is available, according to which the expansion constant associated with the 3 -braid $\sigma_{1} \sigma_{2}^{-1}$ is $\lambda_{1}=\frac{1}{2}(3+\sqrt{5})=2.618 \cdots$.

Here, we show that two particles with small distance are stretched at the rate more than $\lambda_{1}$, which we call the stretching rate. The stretching rate of the chaotic 

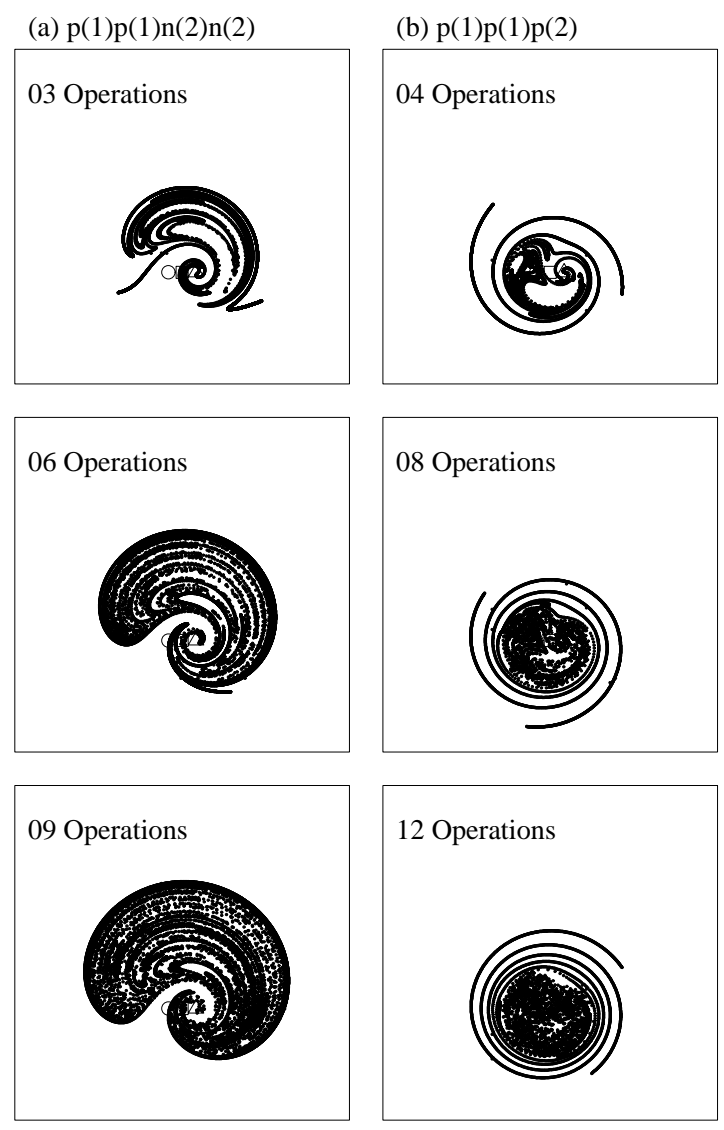

Figure 11: Particle mixings by the operations (a) $p(1) p(1) n(2) n(2)$ and (b) $p(1) p(1) p(2)$. The regularization parameter $\delta$ is 0.6 . 


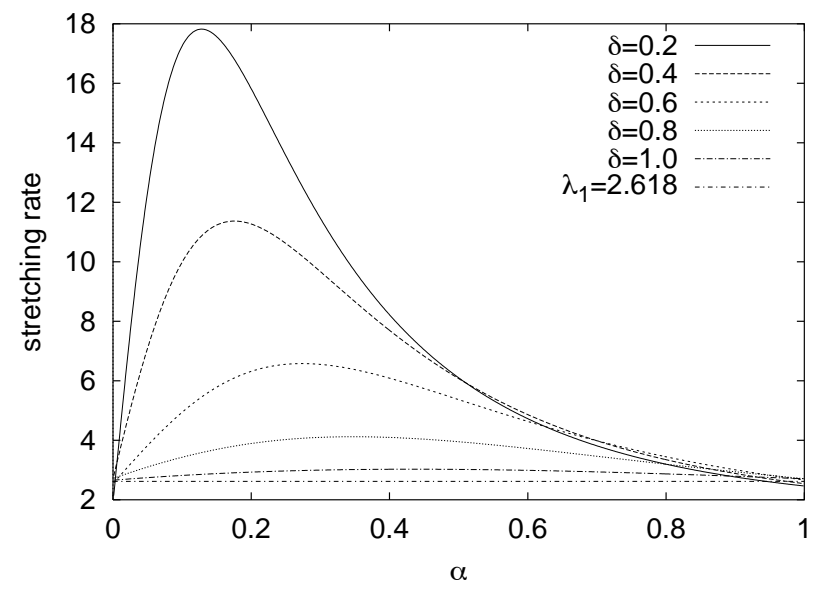

Figure 12: Stretching rate for the particles in the innermost $\bigcirc$-streak by the operation $p(1) n(2)$.

mixing region is computed as follows: We take a very short segment $l(\alpha)$ in the neighborhood of $\bigcirc$, which is parametrized by $0 \leq \alpha \leq 1$, so that the homeomorphism $F \equiv \widetilde{F}_{p(1)} \circ \widetilde{F}_{n(2)}$ maps the line segment $l(\alpha)$ into the innermost $\bigcirc$ streak. Then, we discretize the parameter $\alpha$ by large $M$ points so that $\mid l\left(\alpha_{i+1}\right)-$ $l\left(\alpha_{i}\right) \mid\left(\equiv \epsilon_{i}\right)<1.0 \times 10^{-4}$ for $\alpha_{i}=\frac{i}{M}$. Thus we calculate the stretching rate by $\left|F\left(l\left(\alpha_{i+1}\right)\right)-F\left(l\left(\alpha_{i}\right)\right)\right| / \epsilon_{i}$. Figure 12 shows the stretching rate for various $\delta$, which indicates that the stretching rate is greater than the theoretically estimated expansion constant $\lambda_{1}$. This means that the chaotic mixing region by $p(1) n(2)$ and the topological chaotic mixing region forced by $\sigma_{1} \sigma_{2}^{-1}$ are similar quantitatively.

We compute the stretching rate for another process $p(1) p(1) n(2) n(2)$, whose corresponding braid $\sigma_{1} \sigma_{1} \sigma_{2}^{-1} \sigma_{2}^{-1}$ is $\mathrm{pA}$. The expansion constant for $\sigma_{1} \sigma_{1} \sigma_{2}^{-1} \sigma_{2}^{-1}$ is given by $\lambda_{2}=3+2 \sqrt{2}=5.828 \cdots$. Figure 13 shows the stretching rate for various $\delta$. The particles in the streak are stretched at the rate greater than $\lambda_{2}$.

\subsubsection{Excessive and incomplete mixing}

In the present imaginary mixing device, we have two unrealistic assumptions: First, the vortex blobs are generated as soon as the agitator begins rotating. Second, the particles stop immediately when the agitator stops. However, in reality, the mixing time could be longer or shorter, because the particles have inertia or it takes a moment for the vortex blob to be generated by the agitator.

In order to see that the chaotic mixing is generically observed in more practical situations, we compute the particle mixing by the agitators with excessive mixing time $T^{*}>T$ and incomplete mixing time $T_{*}<T$. Figure 14 shows that the particle mixings by the excessive $p(1) n(2)$ for $\frac{T^{*}}{T}=1.2$ and by the incomplete $p(1) n(2)$ for $\frac{T_{*}}{T}=0.8$. The similar chaotic horseshoe-like regions like in Figure 1 are observed in both cases.

Since the pseudo-Anosov maps are essentially the same as the Anosov diffeomorophisms that are structurally stable, if the topological chaos for $p(1) n(2)$ really appears in the neighborhood of the vortex blobs, then the similar chaotic region to that for $p(1) n(2)$ should be observed under the perturbation of the map. The above numerical experiments show that the strip structure of streaks of the chaotic region is preserved under the the perturbation of the map in terms of the mixing time. 


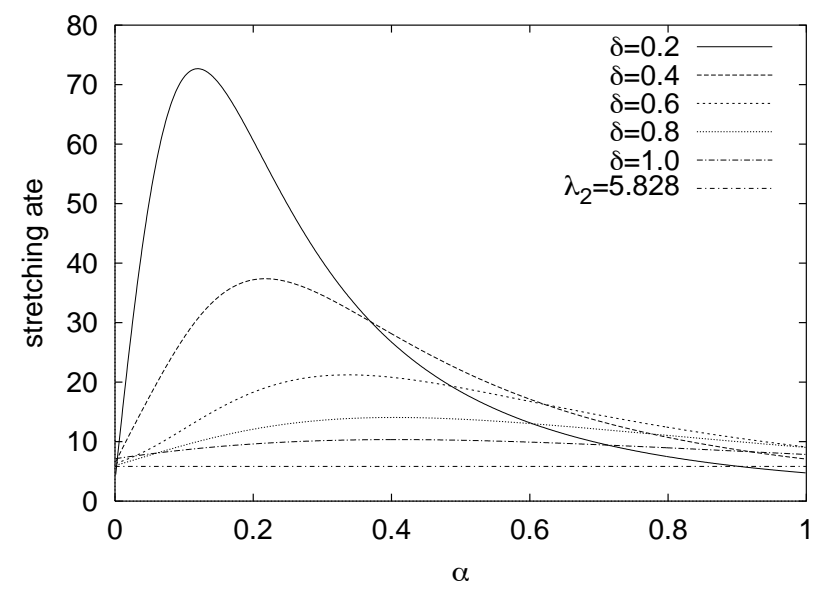

Figure 13: Stretching rate for the particles in the innermost $\bigcirc$-streak by the operation $p(1) p(1) n(2) n(2)$.

(a) excessive mixing
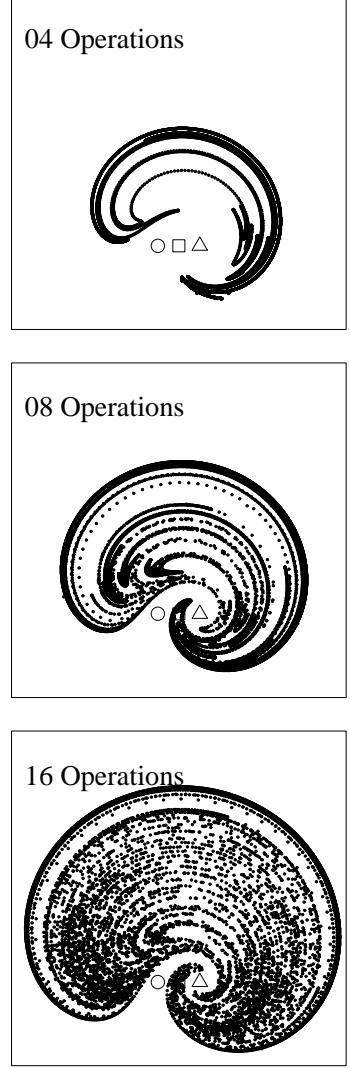

(b) incomplete mixing

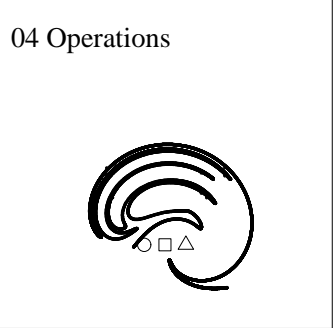

08 Operations
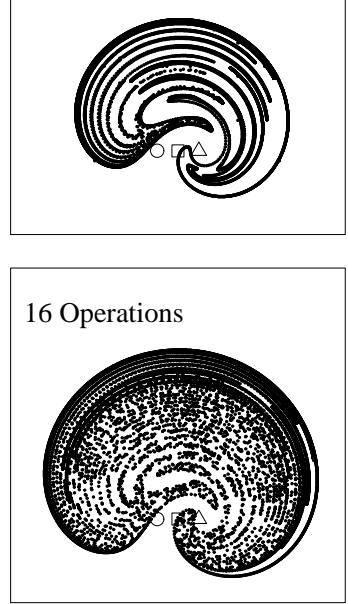

Figure 14: (a) Particle mixing by the excessive operation $p(1) n(2)$. (b) Particle mixing by the incomplete operation $p(1) n(2)$. 


\section{Conclusion}

We have investigated numerically the particle mixing when the two agitators are operated alternatively. Each agitator generates the vortex blob, which is the regularized vortex point, and it stirs the passive particles. Since the particles mixed by the blinking vortex model show the chaotic behavior due to the homoclinic chaos, it is mathematically assured that the topological chaos is embedded in the chaotic mixing region. The conjecture of the present study is that the property of the topological chaos embedded in the neighborhood of the vortex blobs is determined by the Thurston-Nielsen type (periodic, pA, reducible) of the braid assigned to the operation; For the operation $p(1) n(2)$ whose assigned braid is $\mathrm{pA}$, a horseshoe-like structure forced by the pA braid dominates the chaotic region in the neighborhood of the vortex blobs. We have shown some numerical evidences implying that the topological chaos appears dominantly in the neighborhood of the vortex blobs; In Section 4.2.2, any two points in the chaotic region are stretched at the rate more than the expansion constant for the assigned pA braid. In Section 4.2.3, the chaotic region with a horseshoe-like structure is topologically preserved under the perturbation of the map.

Since the chaotic region is connected by the dipole-like return orbits, the structure allows all the particles in the whole plane to move chaotically. Hence, the present study indicates that with the help of the T-N theory, we are able to construct the blinking vortex model that makes all the particles in the whole plane mix efficiently and globally.

On the other hand, for the operation $p(1) p(2)$ whose assigned braid is periodic, the non-uniform chaotic mixing region with the island structures appears in the neighborhood of the vortex blobs, which is surrounded by the laminar rotational orbits. Since the chaotic region is bounded by the rotational orbits, it is hard for all the particles to spread in the plane. In theory, since the $p(1) p(2)$ operation also induces the chaotic advevtion due to the homoclinic chaos, there exists a chaotic invariant set somewhere in the chaotic region forced by the $\mathrm{pA}$ braid, which is not associated with the operation of the blinking vortices. However, the size of the invariant is so small that it hardly contribute to the global chaotic mixing of the particles.

Let us finally remark that the blinking vortex model studied by Aref [1] and Ottino [14] corresponds to the operations assigned to the periodic braid. Hence, the topological chaos is not dominant in their mixing process. Consequently, the particles spread non-uniformly and the chaotic advection occurs on the restricted region in the neighborhood of the blinking vortices. Therefore, all the particles in the plane are hardly mixed chaotically for the blinking vortex model device by Aref and Ottino.

\section{Acknowledgements}

This work is partially supported by Ministry of Education, Science, Sports and Culture, Grand-in-Aid for Young Scientists (B), \#14740069, 2004, and Grand-inAid for formation of COE in Hokkaido University and Kyoto University. 


\section{References}

[1] H. Aref, Stirring by chaotic advection, J. Fluid Mech. 143, (1984) 1-21.

[2] H. Aref ,The development of chaotic advection, Phys. Fluids 14, (2002) 13151325.

[3] M. Bestivina and M.Handel, Train-Tracks for surface homeomorphisms, Topology 34 (1), (1994) 109-140.

[4] P. Boyland, H. Aref and M. Stremler, Topological fluid mechanics of stirring, J. Fluid Mech. 403, (2000) 277-304.

[5] P. Boyland. Topological methods in surface dynamics. Topology Appl. 58 (3), (1994) 223-298.

[6] R.E. Caflisch and J. Lowengrub, Convergence of the vortex method for vortex sheets, SIAM J. Numer. Anal. 26, (1989) 1060-1080.

[7] A. Casson and S. Bleiler, Automorphisms of surfaces after Nielsen and Thurston, London Math. Soc. Stud. Texts 9, Cambridge University Press (1988).

[8] M. D. Finn, S. M. Cox and H. M. Byrne, Topological chaos in inviscid and viscous mixers, J. Fluid Mech. 493, (2003) 345-361.

[9] J. M. Gambaudo, S. Van Strien and C. Tresser, The periodic orbit structure of orientation preserving diffeomorphisms on $D^{2}$ with topological entropy zero, Ann. Inst. H. Poincaré Phys. Theor. 50(3), (1989) 335-356.

[10] T. Hall, the software available at http://www.liv.ac.uk/maths/PURE/MIN_SET/CONTENT/members/T_Hall.html.

[11] M. Handel, Global shadowing of pseudo-Anosov homeomorphisms, Ergodic Theory Dynam. Systems 5 (3), (1985) 373-377.

[12] R. Krasny, Desingularization of periodic vortex sheet roll-up, J. Comput. Phys. 69, (1986) 292-313.

[13] M. Nitsche and R. Krasny, A numerical study of vortex ring formation at the edge of a circular tube, J. Fluid Mech. 276, (1994) 139-161.

[14] J. M. Ottino, The kinematics of mixing: stretching, chaos, and transport, (Cambridge Univ. Press, Cambridge, 1989).

[15] W. Thurston, On the geometry and dynamics of diffeomorphims of surfaces, Bull. Am. Math. Soc. 19 (2), (1988) 417-431.

[16] A. Vikhansky, Simulation of topological chaos in laminar flows, Chaos 14 (1), (2004) 14-22. 